\title{
Analysis and Reduction of Capacitive Coupling Noise in High-Speed VLSI Circuits
}

\author{
Payam Heydari and Massoud Pedram \\ Department of Electrical Engineering-Systems \\ University of Southern California \\ Los Angeles, CA 90089 \\ E-mail: \{payam,massoud $\} @$ sahand.usc.edu
}

\begin{abstract}
Scaling the minimum feature size of VLSI circuits to sub-quarter micron and the clock frequency to $2 \mathrm{GHz}$ has caused the crosstalk noise to become a serious problem, degrading the performance and reliability of high speed integrated circuits. This paper presents an efficient method for computing the capacitive crosstalk in sub-quarter micron VLSI circuits. In particular, we provide closed-form expressions for the peak amplitude, the pulse width, and the time-domain waveform of the crosstalk noise. Experiments show that our analytical predictions are at least two times better than the previous models in terms of the prediction accuracy. More precisely, experimental results show that the maximum error of our predictions is less than $10 \%$ while the average error is only $4 \%$. Finally, based on the proposed analytical models, we discuss the effects of transistor sizing and buffering on crosstalk noise reduction in VLSI circuits.
\end{abstract}

\section{INTRODUCTION}

Rapid advances in VLSI technology has enabled us to reduce the minimum feature sizes to sub-quarter microns and the switching times to tens of picoseconds or even less. Unfortunately, this comes at a cost. The digital circuits have become subject to the same type problem that analog integrated circuits have been affected by since their inception. That problem is noise. Although the device noise sources (i.e shot noise, flicker noise, thermal noise) are still not an issue in the performance of digital circuits, external noise sources (i.e. crosstalk, power/ground bounce, substrate noise) significantly degrade the performance and the reliability of digital integrated circuits. These external noise sources are mostly due to the fact that on-chip interconnects act like transmission lines and that the neighboring wires exert electric and magnetic couplings on each other. Among the various external noise sources, problems related to the on-chip capacitive crosstalk are particularly important. Because the thickness of the wires is not scaled down by as much as the width of the wires and because the wires are packed ever closer to each other, the interwire coupling capacitances become larger, the ratio between the coupling capacitance and the total capacitance increases, and as a result the capacitive coupling noise increases. High-speed digital circuits heavily use the dynamic logic family. Dynamic circuits with their two phase of operations are more susceptible to this kind of noise compared to the static logic.

Various techniques can be utilized to estimate the crosstalk noise. The most accurate but very time consuming approach is to use HSPICE simulation. For example, our experiments show that simulating a small circuit (e.g., a collection of five coupled lossy transmission lines) with HSPICE takes almost three minutes on a $866 \mathrm{MHz}$ Intel processor. Since the interconnects are modeled as linear time-invariant systems, model reduction techniques $[1][2][3][4][5]$ can be utilized to reduce the computational complexity. Model reduction techniques, although helpful, do not adequately solve the problem of long computation times. In addition, these techniques do not provide any insight to the circuit designers as how to modify the circuit structures in order to reduce the crosstalk noise.

Deriving simple closed-form expressions that can predict the noise behavior is more desirable compared to running a simulation tool. This is especially true during the early stages of the design process when one cannot afford to simulate a large number of possible circuit structures and layout solutions. Consequently, a number of researchers have tackled this problem. Vittal et al in [6] provides bounds for the crosstalk noise using a lumped RC model. This work ignores the interconnect resistance. Later on, the same authors in paper [7], use the geometrical properties of the crosstalk noise to obtain expressions for the peak amplitude of the noise as well as the noise pulse width. Their techniques can handle arbitrary input signals. Devgan in [8], proposes a simple yet clever approach to find an upper bound for crosstalk noise. The author himself mentions that his model exhibits a large error when the signals are fast and the rise and fall times are short. Unfortunately, this latter scenario occurs frequently when practical values of the interconnect parasitics and signal frequencies are used. We have observed that the percentage of the estimated error in such cases can be as much as $60 \%$. In addition, Paper [8] does not predict the noise pulse width. Knowing the noise pulse width is important because, in general, the noise margin of a gate depends on both the noise amplitude and pulse width.

In this paper we propose a new crosstalk noise metric that is capable of predicting the noise amplitude and noise pulse width of an RC interconnect as well as an overdamped RLC interconnect. Our noise metric has a closed form expression that clearly specifies the dependency of the noise on the aggressors and victim line circuit parameters as well as the rise/fall times. We then use our metric in two commonly-used noise reduction techniques to calculate relevant characteristics of the line drivers and buffers.

The remainder of the paper is organized as follows. In section 2 , capacitive coupling is reviewed and through experimental results, it is shown that the inductive coupling on chip is negligible for local wiring clocked at a target frequency of $1 \mathrm{GHz}$. After a brief description of Devgan's metric, we introduce our noise metric. We compare our metric with results of Vittal [7] and Devgan [8] in a series of detailed experiments. Next, in section 3 we introduce some techniques to reduce the crosstalk, and we will show how our metric can be suitably fitted to these noise reduction techniques. Section 4 has our concluding remarks.

\section{Capacitive coupling}

As the circuit speeds increase, the effects of on-chip crosstalk noise become more pronounced. Fig. 1 . shows $N$ neighboring wires. The high frequency operation of VLSI circuits causes the on-chip wires to exhibit transmission line effects, and hence we have electrical and magnetic couplings between any pair of wires. These electric and magnetic couplings reshape the signal waveforms and potentially induce delay in the signals traveling through the lines.

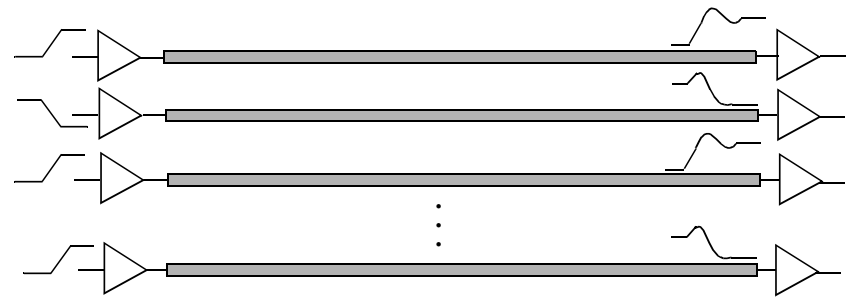

Fig. 1. Circuit schematic of $N$ on-chip interconnects

Figures 2 and 3 depict five microstrip lines powered by five CMOS drivers along with their distributed RLC circuit model. The geometrical parameters of the lines and device sizes are shown in the figures. The wire lines and the shieldings are all in copper. The input to the first, the second, and the fourth lines are periodic square waveforms with non-zero rise and fall-times of 80 psec. The third and the fifth lines are held steady with a high input voltage at the input of the first driver and the fifth driver. The cycle-time is 2nsec. By running HSPICE on this circuit, we extract the following capacitance $\mathbf{C}$, inductance $\mathbf{L}$, and resistance $\mathbf{R}$ matrices per unit length.

Comparing the values of the matrix elements reveals that the coupling capacitance of non-adjacent wires is very small compared to the adjacent coupling capacitances. This is obviously not the case for the mutual inductances. With these observations, an effective model for coupled interconnects can be constructed by assuming that each line is capacitively coupled only to its immediate neighboring wires, whereas it is inductively coupled to every other wire. However, as will be seen later, our noise metric also handles the capacitive coupling effect of non-adjacent wires. 


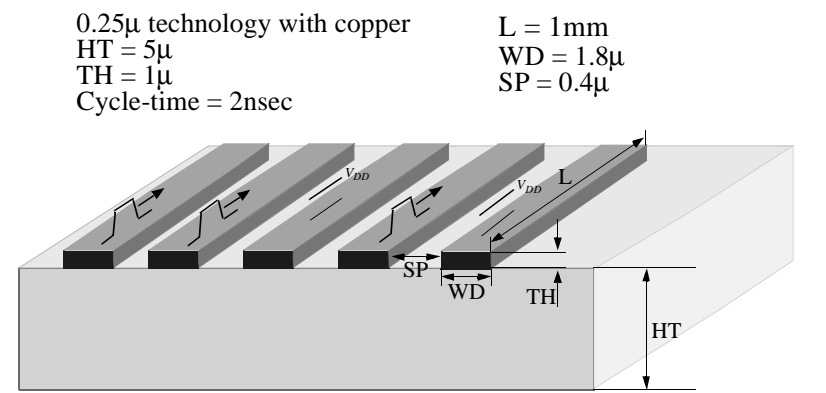

Fig. 2. Five parallel microstrip lines in $0.25 \mu$ CMOS technology

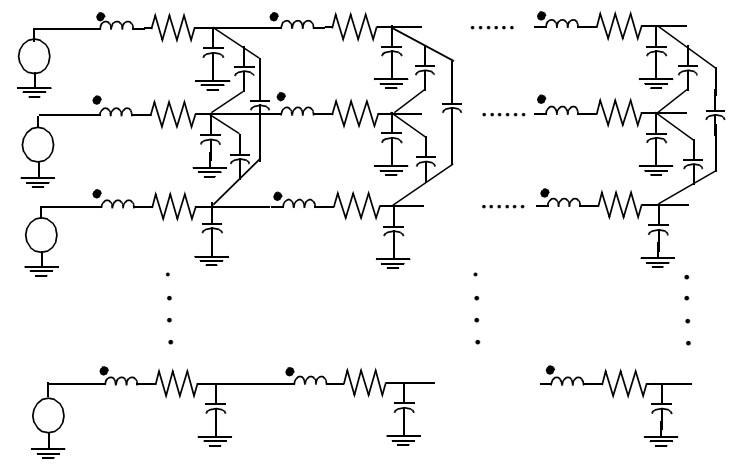

Fig. 3. Circuit schematic of $\mathrm{N}$ interconnects that area eletromagnetically coupled to each other

$$
\begin{aligned}
\mathbf{C} & =\left[\begin{array}{ccccc}
81.32 & 47.87 & 1.7 & 0.844 & 0.579 \\
47.87 & 118.7 & 46.95 & 1.25 & 0.845 \\
1.7 & 46.95 & 118.75 & 46.95 & 1.7 \\
0.844 & 1.25 & 46.95 & 118.7 & 26.46 \\
0.579 & 0.845 & 1.7 & 47.87 & 81.32
\end{array}\right](\mathrm{pF} / \mathrm{m}) \\
\mathbf{L} & =\left[\begin{array}{lllll}
479.29 & 325.08 & 232.49 & 175.07 & 140.4 \\
325.08 & 434.3 & 300.6 & 221.25 & 175.07 \\
232.49 & 300.6 & 423.8 & 300.6 & 232.49 \\
175.07 & 221.25 & 300.6 & 434.3 & 325.08 \\
140.4 & 175.05 & 232.49 & 325.08 & 479.27
\end{array}\right](\mathrm{nH} / \mathrm{m}) \\
\mathbf{R} & =\left[\begin{array}{cccccc}
11.47 & 9.56 & 9.56 & 9.56 & 9.56 \\
9.56 & 11.47 & 9.56 & 9.56 & 9.56 \\
9.56 & 9.56 & 11.47 & 9.56 & 9.56 \\
9.56 & 9.56 & 9.56 & 11.47 & 9.56 \\
9.56 & 9.56 & 9.56 & 9.56 & 11.47
\end{array}\right](\mathrm{K} \Omega / m)
\end{aligned}
$$

Fig. 4. Capacitance, inductance and resistance matrices of Fig. 2

In general, if the reactance of the interconnect inductance at the clock frequency is negligible compared to the interconnect resistance, the interconnect can simply be represented by a distributed $\mathrm{RC}$ circuit. This condition is normally met by short local wirings on a chip. To examine the above condition on our particular experiment, we first compute the interconnect parasitics using the geometrical parameters of the interconnects. The microstrip capacitance per inch of a microscope line is [9]:

$$
C_{\text {line }}=\frac{1.41 \varepsilon_{\text {eff }}}{\ln \left(\frac{8 h}{w}+\frac{w}{4 h}\right)} \quad \text { where: } \varepsilon_{\text {eff }}=\left(\frac{\varepsilon_{r}+1}{2}\right)+\left(\frac{\varepsilon_{r}-1}{2}\right)\left(1+\frac{10 h}{w}\right)^{-\frac{1}{2}}
$$

where $w$ is the conductor width, and $h$ is the dielectric thickness. The inductance per length of an interconnect, when the return path is the reference plane would be [9]:

$$
L_{\text {line }}=\frac{7.3 \varepsilon_{r}}{C_{\text {line }}} \quad\left[\mathrm{nH} / \text { inch with } C_{L} \text { in } \mathrm{PF} / \text { inch }\right]
$$

Substituting the actual geometrical values from Fig. 2. in the inductance and capacitance equations yields the following values:

$$
C_{\text {line }}=0.216 \mathrm{pF}, L_{\text {line }}=1.18 \mathrm{nH}, R_{\text {line }}=49.71 \Omega
$$

For the interconnect circuit in Fig. 2. the resistance value is more than ten times greater than the impedance represented by the inductance at 2 nsec clock cycle time. Therefore, the distributed $\mathrm{RC}$ circuit representation is used instead of the more accurate distributed RLC representation. To verify this simplification, the circuit shown in Fig. 2. is simulated with HSPICE. First, the distributed RLC circuit that was extracted from the HSPICE output file is used. The simulation is run on this circuit to determine the noise waveform. In the next step, inductances are set to zero, and the simulation is rerun on this new circuit. Fig. 5. shows the results of these simulation on the circuit shown in Fig. 2. From Fig. 5., the noise waveforms of two circuits are the same and we cannot distinguish these two waveforms in the figure. The absence of any ringing at the output voltage of the interconnect (with a 2 nsec clock cycle time) validates the assumption of modeling the interconnect with distributed RC circuits only.
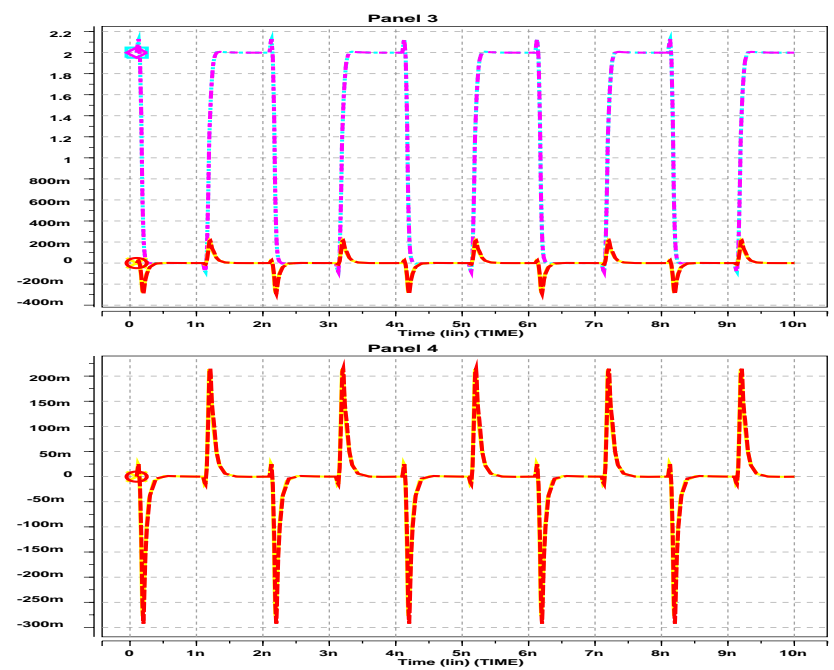

Fig. 5. Comparison between the distributed RC circuit model and RLC model of Fig. 2 using the HSPICE simulation

From Fig. 5. we can see that for local wires the on-chip capacitive coupling is more pronounced than the on-chip inductive coupling. Therefore, in this paper, we focus on the capacitive coupling noise problem.

Our goal is to develop a circuit model to predict the capacitive coupling for on-chip coupled interconnects and thereby derive a closed-form expression for the crosstalk noise. We start our analysis by reviewing the derivation of Devgan's metric and its drawback for crosstalk noise estimation of RC circuits. For a more comprehensive explanation of this metric, please refer to [8].

\subsection{Devgan's metric for crosstalk noise estimation}

Consider two capacitively coupled RC networks as shown in Fig. 6 . One RC ladder network (called the aggressor net) is driven by a flattened ramp voltage whereas the second RC ladder (called the victim net) is quiet. For this circuit, the node voltage vector at the victim net, $\mathbf{V}_{2} \in \Re^{N \times 1}$, is related to the voltage vector at the aggressor net, $\mathbf{V}_{1} \in \Re^{N \times 1}$, through the following equation:
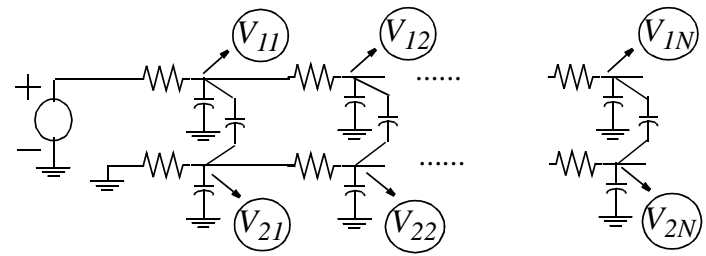

Fig. 6. Circuit schematic of capacitively coupled aggressor and victim nets 


$$
\begin{gathered}
{\left[\left(s \mathbf{C}_{2}-\mathbf{A}_{22}\right)-s \mathbf{C}_{c}\left(s \mathbf{C}_{1}-\mathbf{A}_{11}\right)^{-1} s \mathbf{C}_{c}\right] \mathbf{V}_{2}} \\
=-s \mathbf{C}_{c}\left(s \mathbf{C}_{1}-\mathbf{A}_{11}\right)^{-1} \mathbf{B}_{1} \mathbf{V}_{s}
\end{gathered}
$$

where $\mathbf{C}_{i}=\operatorname{diag}\left(C_{i}+C_{c}\right)$ for $i=1,2$, and $\mathbf{C}_{c}=\operatorname{diag}\left(-C_{c}\right) . \mathbf{A}_{11}$ and $\mathbf{A}_{22}$ represent the equivalent node resistance matrices of the aggressor net and the victim net, respectively. Given an infinite ramp input, the node voltages at the victim node monotonically go toward their final values. Hence, the voltage values at $t=\infty$ are indeed the largest possible values of node voltages at the victim net. The final value theorem is utilized to determine the steadystate values of node voltages at the victim net. The result is:

$$
\mathbf{V}_{2, \text { max }}=-\mathbf{A}_{22}^{-1} \mathbf{C}_{c} \mathbf{A}_{11}^{-1} \mathbf{B}_{1} \frac{V_{D D}}{t_{r}}
$$

where $t_{r}$ is the rise-time of the signal. For simplicity, it is assumed that the rise and fall times are equal. Note that this result is valid only if the driving voltages of the interconnects are infinite ramps. This is a critical assumption that seriously limits the accuracy for capacitive crosstalk estimation. In practice, the actual driving voltages of the interconnects are saturated ramp inputs rather than infinite ramps. This means that the node voltages at the victim net reach their peak value at $t=t_{r}$. This peak value is obviously different from the steady-state value under the infinite ramp input, and the error between these two values can be quite large if the risetime of the input is fast.

To better understand the shortcoming of this approach, consider two second-order RC circuits with two floating capacitances connecting the corresponding nodes of these two sections as shown in Fig. 7.

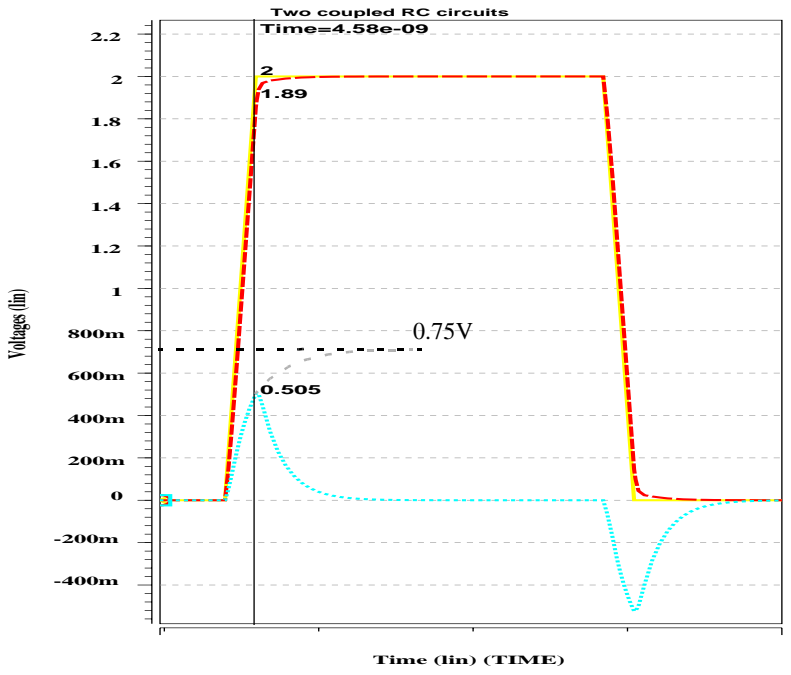

Fig. $\%$ The output voltage and the crosstalk of two coupled secondorder RC circuits. $C_{1}=60 f F, C_{2}=120 f F, R_{2}=100, R_{1}=20, C_{c}=$ 100fF, $\mathrm{t}_{\mathrm{r}}=0.08 \mathrm{~ns}$

According to the HSPICE simulation, the reported peak value of voltage $V_{22}$ is $0.505 \mathrm{~V}$. Devgan's metric for two coupled RC sections yields the following results:

$$
\begin{gathered}
V_{21, \text { max }}=2\left(R_{2}+R_{s_{2}}\right) C_{c} \frac{V_{D D}}{t_{r}} \\
V_{22, \text { max }}=\left(2 R_{2}+3 R_{s_{2}}\right) C_{c} \frac{V_{D D}}{t_{r}}
\end{gathered}
$$

Using Eq. (6), $V_{22, \max }$ is $0.75 \mathrm{~V}$. The estimated error is $48.5 \%$. Since the rise-time is small, the crosstalk waveform rolls down quickly, and as a result, the error becomes unacceptably large. For cases where the rise-time is large compared to the interconnect delays, Devgan's metric can accurately predict the peak value. Unfortunately, cases in which the estimations are accurate (i.e., the slow slew-time for the pulses), are unimportant from a circuit performance viewpoint. The reason is that the peak value of the crosstalk is inversely proportional to the input rise-time. For slow slew waveforms, the crosstalk also has a small peak value and thus has little effect on the circuit delay and logic failure rate.

In the next section we derive a new, more accurate, noise metric and compare our results with Devgan's results and with HSPICE simulations.

\subsection{A new metric for crosstalk noise estimation}

Examining the HSPICE results reported in Fig. 7 helps us identify one source of inaccuracy in Devgan's metric. The large error in this example comes from the fact that the time constants of the exponentially rising portions of victim node voltages are comparable to (or larger than) the input rise time. The actual peak value of the crosstalk occurs at $t=t_{r}$. To compute this peak value, we recognize that the capacitive crosstalk noise at every node of the victim net is a rising exponential function in the input transient interval. The actual peak value of the crosstalk noise at every node of the victim net is the value of the corresponding rising exponential function at $t=t_{r}$ where the steady-state value of this exponential function is determined by Devgan's metric.

$$
V_{2 j, \text { max }}=V_{2 j, s s}\left(1-\exp \left(-t_{r} / \tau_{d_{j}}\right)\right) \quad \text { for } j=1,2, \ldots, N
$$

where $\tau_{d_{j}}$ is the time constant of the $j$-th node voltage in the victim net, and $V_{2 j, s s}$ is the steady state value of the crosstalk noise as calculated from Devgan's metric. Each node in the victim net sees two capacitances: a grounding capacitance, $C_{2 j}$, and a floating coupled capacitance, $C_{c j}$. The time constant at each victim node is proportional to the time constants seen across each of these two capacitances. To accurately estimate this time constant, we first construct an equivalent circuit consisting of $C_{2 j}, C_{c j}$, and the equivalent resistances seen across these two capacitances with all other capacitances being replaced with open circuits. This circuit model is shown in Fig. 8.

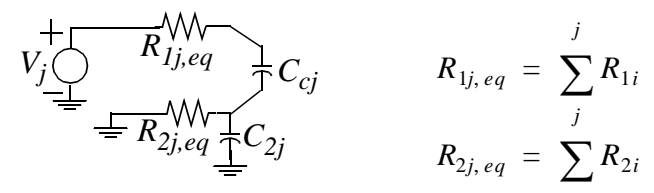

Fig. 8. The equivalent circuit for computing the time constant of the $j$ th node of the victim net

The characteristic polynomial of this second-order transfer function is:

$$
\Lambda_{j}(s)=R_{1 j, e q} R_{2 j, e q} C_{2 j} C_{c j} s^{2}+\left[\left(R_{1 j, e q}+R_{2 j, e q}\right) C_{c j}+R_{2 j, e q} C_{2 j}\right] s+1
$$

The time constant of this second-order circuit, which is roughly the $3-\mathrm{dB}$ bandwidth of the system transfer function, is equal to the coefficient of the first-order term. It should be noted that the input voltage source is assumed to be a unit step function. This is obviously not the case for the coupled RC circuits. The input voltage to the $j$-th node of the aggressor itself experiences an $\mathrm{RC}$ delay due to the existing RC path from the input to the $j$-th node. This RC delay also needs to be accounted for. In addition, for RC circuits with orders greater than one, the initial slope of the step and ramp responses are zero. This zero initial slope leads to an increase in the circuit delay. Considering all these effects in our formulations yields the following expression for $\tau_{d_{j}}$.

$$
\tau_{d_{j}}=\Gamma\left[\left(R_{1 j, e q}+R_{2 j, e q}\right) C_{c j}+R_{2 j, e q} C_{2 j}+\tau_{a_{j}}\right]
$$

where $\tau_{a_{i}}$ is:

$$
\tau_{a_{j}}=\sum\left[R_{1 k, e q}\left(C_{c k}+C_{1 k}\right)+R_{2 k, e q} C_{2 k}\right]+R_{1 j, e q}\left(C_{c j}+C_{1 j}\right)
$$

and $\Gamma$ is a constant factor that is for the delay increase due to the nonzero finite input slope. Its value is in the range [1.05,1.1]. Throughout our analysis we will assume that $\Gamma=1.07$. As can be seen, the peak amplitude of the crosstalk is easily obtained by these calculations. To measure the level of accuracy that can be obtained by our metric, the peak crosstalk noise of two coupled second-order RC sections with different values for the input risetime and $\mathrm{RC}$ values is computed and the results are compared with 
those obtained by HSPICE simulation as well as by Devgan's metric. The supply voltage is $V_{D D}=2 \mathrm{~V}$, and the cycle-time is $2 n s e c$. Table 1 contains the results of these comparisons.

To verify the accuracy of our approach on multistage RC stages and also to compare with other expressions proposed in [7] and [8], we set up a set of experiments on a two-line structure in $0.25 \mu$ CMOS technology. The coupling lengths of the adjacent interconnects are varied from $100 \mu \mathrm{m}$ to $2 \mathrm{~mm}$. Results are compared for a range of rise-times varying between 30ps and 200ps. Table 2 contains the result of these comparisons. The mean and maximum errors are reported in Table 3 . These tables demonstrate the higher accuracy of our approach compared to the two previous noise expressions reported in papers [7] and [8].

The noise susceptibility of logic gates depends not only on the peak amplitude of the crosstalk noise, but also its duration. For example, digital circuits can often tolerate (and indeed filter out) spike-like crosstalk noise with a large peak amplitude and very small noise pulse width. Furthermore, in static logic circuits, the peak amplitude of crosstalk does not result in loss of signal values. Instead, it tends to cause an increase in propagation delay along the victim line (which in turn may cause setup time violation in high-speed circuits). These observations creates the necessity for determining the complete noise waveform.

Given the equivalent time constant and the peak amplitude of the crosstalk, the noise waveform can be calculated by the following equation:

$$
V_{2 j}(t)=\left\{\begin{array}{lr}
V_{2 j, s s}\left(1-\exp \left(-t / \tau_{d_{j}}\right)\right) & 0 \leq t \leq t_{r} \\
V_{2 j, \text { max }} \exp \left(-\left(t-t_{r}\right) / \tau_{d_{j}}\right) & t \geq t_{r}
\end{array} \text { for } j=1,2, \ldots, N\right.
$$

Fig. 9. compares Eq. (11) with HSPICE simulation for a pair of capacitively coupled nets. As one can see, the new metric can accurately predict not only the noise peak amplitude, but also the noise pulse width. Indeed, the effective pulse width is estimated within $5 \%$ error.

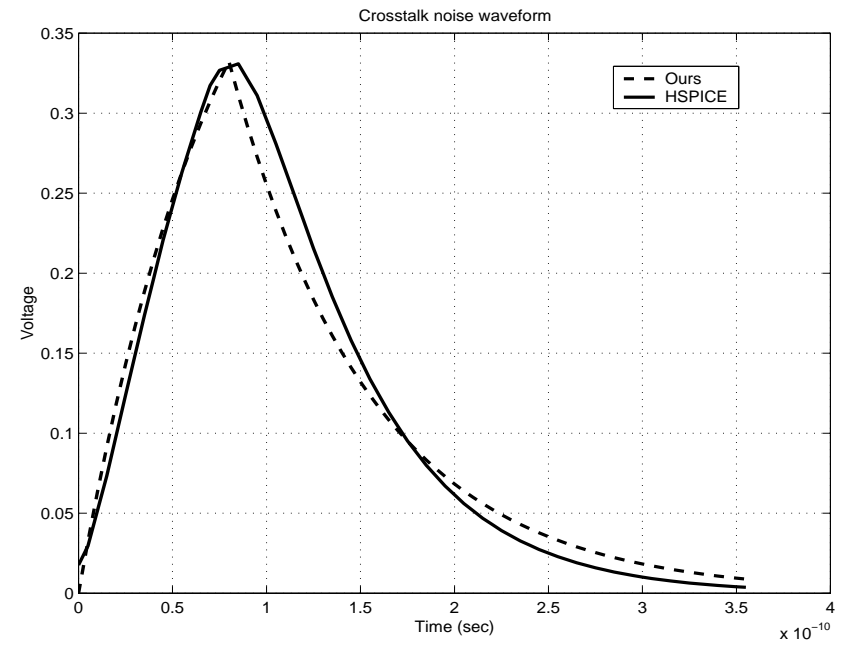

Fig. 9. Crosstalk noise waveforms for two coupled transmission lines.

The line characteristics are given as the last entry of table 2

Fig. 10. shows the change in crosstalk when the input rise time varies from 50ps to $300 \mathrm{ps}$ and all the geometrical parameters are fixed. Comparing HSPICE with our approach confirms that one achieves a high accuracy with our noise metric over a wide range of input rise-times. As expected, for the long rise-times the Devgan's metric accurately predicts the peak amplitude of the noise. Vittals' metric produces higher fidelity results compared to Devgan's: its estimation error remains roughly constant and does not have as large a dynamic range as Devgan's. Our metric is more accurate than the works in papers [7] and [8].

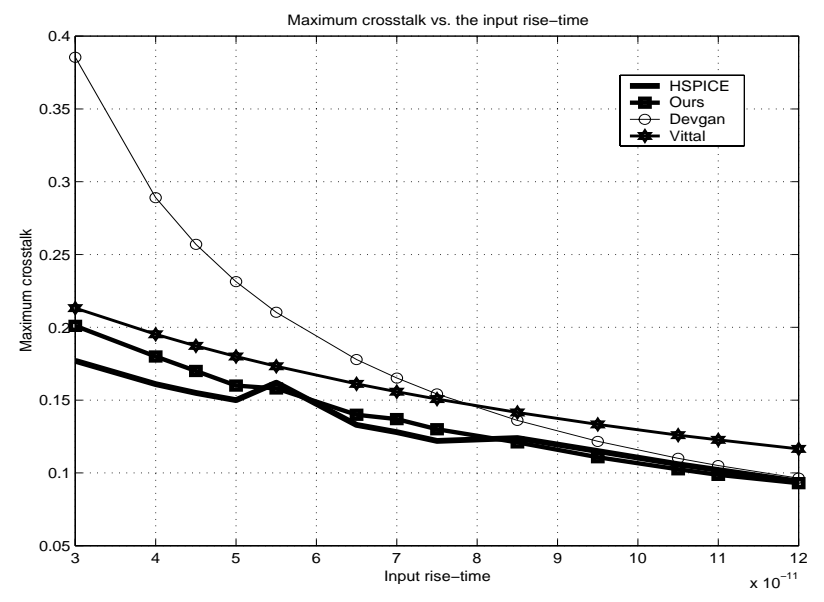

Fig. 10. Maximum crosstalk noise vs. input rise-time

\section{Crosstalk NOISE AND delay ReduCtion}

We use our noise metric to provide some guidelines for reducing the crosstalk peak value and the negative impact of the crosstalk on circuit delay.

\subsection{Transistor sizing of the aggressor driver}

From equations (9) and (10), we realize that reducing the (W/L) ratios of the aggressor net driver increases the time constant, $\tau_{d j}$, and as a consequence, reduces the peak value of the crosstalk noise. Therefore, one might think of scaling down the (W/L) ratio as a tool for crosstalk reduction. This solution should, however, be used cautiously as explained next.

First of all, with the reduction of the (W/L) ratio, the drive capability of the driver also decreases. Therefore, it takes a longer time to actually charge or discharge the load impedance. As a result, this may lead to a delay increase in the circuit. Another problem is that we may decrease the peak amplitude of the crosstalk, but at the same time increase the noise pulse width. We use the noise peak amplitude-pulse width product (AWP) as a figure of merit to determine the usefulness of transistor sizing for crosstalk reduction. The AWP is formally defined as:

$$
A W P=\left(t_{r}+3 \tau_{d_{N}}\right) \cdot V_{x t a l k, \max }
$$

Note that $t_{r}+3 \tau_{d_{N}}$ is the pulse width of the crosstalk noise. The second term, $3 \tau_{d_{N}}$, is the settling time of a growing exponential function. In general, this settling time is three to four times larger than the time constant of the crosstalk voltage, $\tau_{d_{N}}$.We assume that the ratio between the settling time and the time constant of the growing exponential function is three.

Fig. 11. shows the variation of the noise peak value in terms of the (W/L) ratio of the aggressor driver. As expected, the peak amplitude of the crosstalk noise increases with the (W/L) ratio of the aggressor driver. Fig. 12. shows the variations of AWP as a function of (W/L). Although the peak amplitude is an increasing function of the $(\mathrm{W} / \mathrm{L})$ ratio of the aggressor driver, the AWP is not. Therefore if the noise pulse width is as critical to performance as the peak amplitude of the crosstalk, we would increase the (W/L) ratio of the aggressor to get a smaller AWP.

\subsection{Use of Schmitt trigger circuit as buffer}

An effective circuit technique to filter out the crosstalk noise is to do buffer insertion. Alpert et. al in [10] show that buffer insertion is effective for simultaneous optimization of timing and noise. This paper, however, uses the Devgan's metric for formulating the crosstalk noise constraint. As we saw in the previous section, this metric produces a large error for short input rise-times. An obvious improvement would be to use our new metric for capturing the noise constraints. Going further, using Schmitt trigger circuits instead of buffers provides us with the flexibility to adjust the switching threshold voltage according to the direction of the input signal transition as illustrated in Fig. 13. From a circuit modeling 
point of view, the Schmitt trigger operates like a resized inverter with $W_{n}>>W_{p}$ for a low-to-high transition at the input and with $W_{p}>>W_{n}$ for a high-to-low transition at the input. Notice that because of this adjustment to the switching threshold of the Schmitt trigger buffers, these buffers are less susceptible to the crosstalk noise, i.e., they can filter out noise pulses with large AWP.

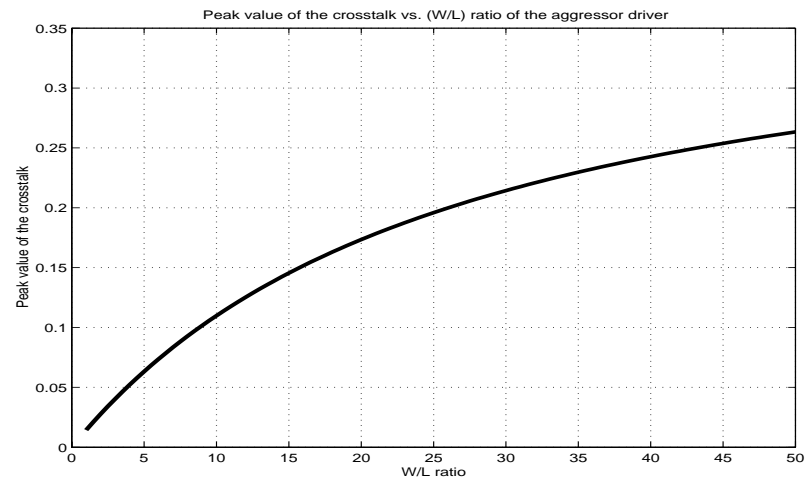

Fig.11. Peak amplitude of the crosstalk vs. the W/L of the aggressor driver

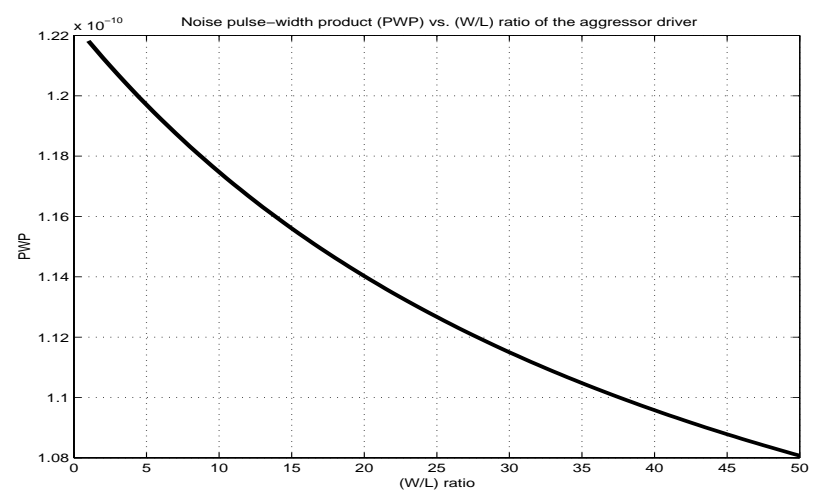

Fig. 12. AWP vs. the W/L of the aggressor driver

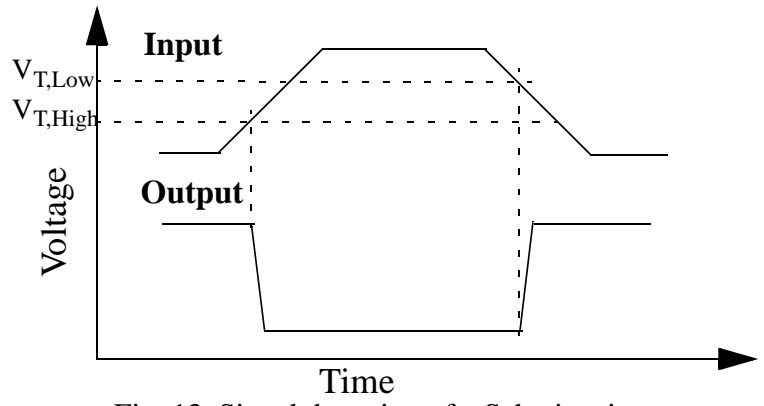

Fig. 13. Signal detection of a Schmitt trigger

To use a convenient circuit structure for the Schmitt trigger we should consider some important facts here:

1. The Schmitt trigger should compensate for the line delay. It should have as high a gain in transition response as an inverter with the corresponding gate size.

2 . The Schmitt trigger should operate correctly in the sub quarter micron CMOS technology with low supply voltage in the range of $1.3 \mathrm{~V}-1.8 \mathrm{~V}$. As a consequence, the circuit structure should not contain stacked transistors. Given the above facts, we use the circuit structure shown in Fig. 14.

The ratio of gate aspect ratios of transistors $M N_{l}$, and $M N_{2}$ are used to define the lower and upper threshold voltage of the Schmitt trigger. The operation of this Schmitt trigger is described next.

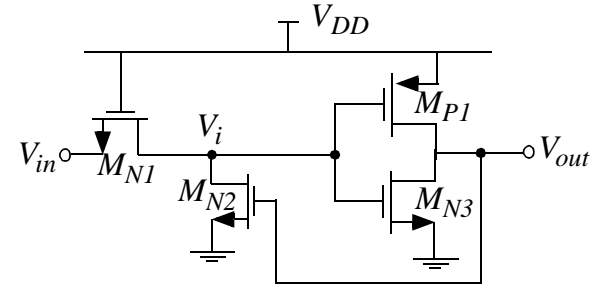

Fig. 14. The Schmitt trigger circuit used for filtering the crosstalk

Suppose that the input is initially low $\left(V_{i n}=O V\right)$. Transistors $M N_{1}$ and $M N_{2}$ are in their linear regions, and the voltage $V_{i}$ is obtained by a resistive voltage division from the input voltage. The output voltage is in the high logic state. As the input voltage increases, voltage $V_{i}$ also increases at a lower rate due to the RC time constant seen at this node. When $V_{i}$ reaches the threshold voltage of the NMOS transistor $M N_{3}$, the logic switching occurs. The positive feedback across the inverter that is produced by $\mathrm{MN}_{2}$ causes a very fast transition time at the output. The threshold voltage for the high-to-low transition at the output is thus equal to:

$$
V_{T H L}=V_{t n}\left(\frac{r_{D S_{2}}+r_{D S_{1}}}{r_{D S_{2}}}\right)
$$

Now for the low-to-high transition at the output the same analysis is done, with the exception of having the threshold voltage of $M P_{1}$ as the point where the low-to-high logic switching occurs. The threshold voltage for the high-to-low transition at the output is thus equal to:

$$
V_{T L H}=V_{D D}-V_{t n, b o d y}-\left|V_{t p}\right|
$$

where $r_{D S_{1}}$ and $r_{D S_{2}}$ are the on-resistance of transistors $M N_{1}$ and $M N_{2}$ respectively. $V_{t n, \text { body }}$ is the threshold voltage of $M N_{1}$. Fig. 15. shows the input and output waveforms of the Schmitt trigger circuit, with the gate aspect ratios depicted in the figure.

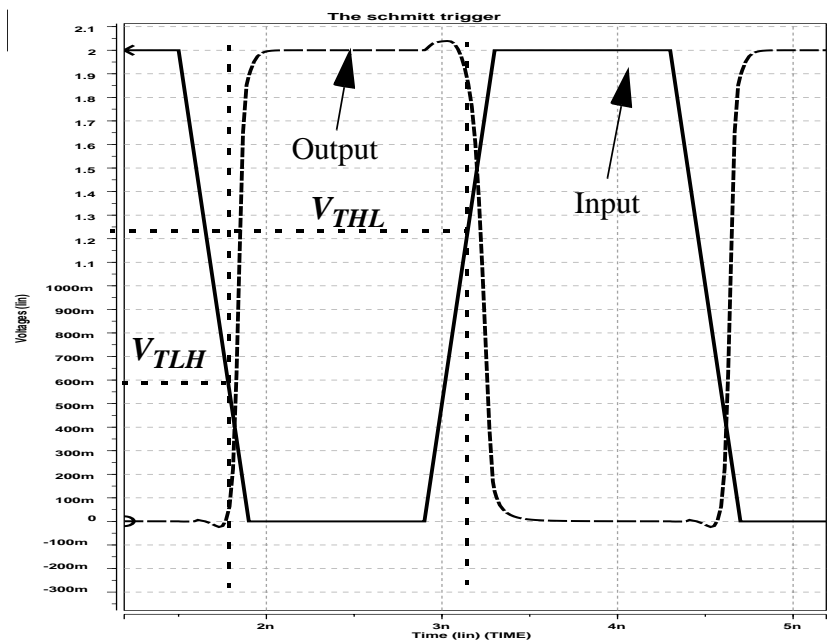

Fig. 15. Input and output waveform of the Schmiit trigger shown in Fig. 14

We use our noise metric to resize the transistors of the Schmitt trigger for noise reduction and delay minimization.

\section{Conclusions}

In this paper, we presented an efficient analysis technique for the capacitive crosstalk computation in sub-quarter micron VLSI interconnects. We derived closed-form expressions for the peak amplitude, the pulse width, and the time-domain waveform of 
Table 1. Comparison of the crosstalk noise computed by HSPICE, paper [8], and our metric

\begin{tabular}{|c|c|c|c|c|c|c|c|c|}
\hline $\begin{array}{l}\mathbf{C}_{1} \\
\mathrm{fF}\end{array}$ & $\begin{array}{l}\mathbf{C}_{2} \\
\mathrm{fF}\end{array}$ & $\mathbf{R}_{2}$ & $\mathbf{R}_{1}$ & $\begin{array}{l}\mathbf{C}_{\mathbf{c}} \\
\mathrm{fF}\end{array}$ & $\begin{array}{l}\mathbf{t}_{\mathbf{r}} \\
\mathrm{ns}\end{array}$ & $\begin{array}{c}\text { HSPICE } \\
\text { volts }\end{array}$ & $\begin{array}{c}\text { Devgan } \\
\text { volts }\end{array}$ & $\begin{array}{l}\text { Ours } \\
\text { volts }\end{array}$ \\
\hline 50 & 60 & 100 & 100 & 30 & 0.05 & 0.216 & 0.36 & 0.229 \\
\hline 60 & 60 & 100 & 100 & 30 & 0.4 & 0.045 & 0.045 & 0.044 \\
\hline 70 & 70 & 300 & 50 & 50 & 0.1 & 0.533 & 0.9 & 0.547 \\
\hline 70 & 60 & 80 & 70 & 50 & 0.3 & 0.08 & 0.08 & 0.08 \\
\hline 100 & 120 & 80 & 70 & 90 & 0.3 & 0.143 & 0.144 & 0.141 \\
\hline 100 & 120 & 80 & 40 & 60 & 0.1 & 0.25 & 0.288 & 0.235 \\
\hline 100 & 120 & 100 & 40 & 60 & 0.09 & 0.291 & 0.4 & 0.311 \\
\hline 100 & 120 & 70 & 30 & 100 & 0.08 & 0.39 & 0.525 & 0.389 \\
\hline$\overline{120}$ & 70 & 30 & 100 & 100 & 0.08 & 0.144 & 0.225 & 0.131 \\
\hline 60 & 120 & 100 & 20 & 100 & 0.08 & 0.505 & 0.75 & 0.53 \\
\hline 80 & 220 & 200 & 90 & 160 & 0.08 & 0.581 & 2.4 & 0.61 \\
\hline 70 & 100 & 60 & 40 & 100 & \begin{tabular}{c|c|}
0.03 \\
\end{tabular} & 0.513 & 1.2 & 0.52 \\
\hline
\end{tabular}

Table 2. The results of simulations on the 2 capacitively coupled transmission lines using star-HSPICE, paper [7], paper [8], and our metric

\begin{tabular}{|c|c|c|c|c|c|c|c|c|c|c|c|c|c|c|}
\hline $\begin{array}{l}c_{1} \\
(p F / m)\end{array}$ & $\begin{array}{l}\mathbf{r}_{1} \\
(\mathbf{k} \Omega / \mathbf{m})\end{array}$ & $\begin{array}{l}\mathbf{r}_{2} \\
(\mathbf{k} \Omega / \mathbf{m})\end{array}$ & $\begin{array}{l}c_{2} \\
(\mathrm{pF} / \mathrm{m})\end{array}$ & $\begin{array}{l}\mathbf{c}_{\mathrm{c}} \\
(\mathrm{pF} / \mathrm{m})\end{array}$ & $\mathbf{R}_{\mathrm{s} 1}$ & $\mathbf{R}_{\mathrm{s} 2}$ & $\begin{array}{l}\mathbf{C}_{\text {out1 }} \\
\text { (pF) }\end{array}$ & $\begin{array}{l}\mathbf{C}_{\text {out2 }} \\
(\mathbf{p F})\end{array}$ & $\begin{array}{l}\mathbf{t}_{\mathbf{r}} \\
\text { nsec }\end{array}$ & $\begin{array}{l}\mathbf{L} \\
(\mathbf{m m})\end{array}$ & $\begin{array}{l}\text { HSPICE } \\
\text { volts }\end{array}$ & $\begin{array}{l}\text { Devgan } \\
\text { volts }\end{array}$ & $\begin{array}{l}\text { Vittal } \\
\text { volts }\end{array}$ & $\begin{array}{l}\text { Ours } \\
\text { volts }\end{array}$ \\
\hline 88.47 & 11.47 & 11.47 & 89.47 & 54.36 & 500 & 150 & 0.3 & 0.05 & 0.03 & 0.1 & $\mathbf{0 . 0 3 3 3}$ & 0.05457 & 0.0333 & 0.0362 \\
\hline 97.87 & 9.55 & 9.55 & 97.87 & 78 & 20 & 40 & 0.3 & 0.1 & 0.03 & 0.8 & 0.129 & 0.1831 & 0.1589 & 0.1377 \\
\hline 120 & 10.2 & 10.2 & 100 & 82 & 90 & 100 & 0.4 & 0.06 & 0.1 & 0.9 & 0.112 & 0.1547 & 0.1206 & 0.11 \\
\hline 151 & 12 & 12 & 120 & 100 & 40 & 0.08 & 0.3 & 0.07 & 0.08 & 1 & 0.176 & 0.2158 & 0.2026 & 0.1798 \\
\hline 200 & 17 & 17 & 190 & 155 & 20 & 10 & 0.3 & 0.05 & 0.07 & 1.6 & 0.129 & 0.172 & 0.162 & 0.133 \\
\hline 235 & 20 & 20 & 220 & 200 & 15 & 20 & 0.05 & 0.1 & 0.12 & 2 & 0.236 & 0.2733 & 0.2546 & 0.2302 \\
\hline 235 & 20 & 20 & 220 & 200 & 20 & 30 & 0.07 & 0.08 & 0.08 & 2 & 0.321 & 0.51 & 0.3325 & 0.3323 \\
\hline
\end{tabular}

Table 3. Error comparison for three noise metrics

\begin{tabular}{|c|c|c|c|}
\hline & $\begin{array}{c}\text { \% Error } \\
\text { Devgan's }\end{array}$ & $\begin{array}{l}\text { \% Error } \\
\text { Vittal's }\end{array}$ & $\begin{array}{c}\text { \% Error } \\
\text { Ours }\end{array}$ \\
\hline & 63.4 & 21.6 & 8.7 \\
\hline & 52 & 20.1 & 6.22 \\
\hline & 41 & 23.2 & 6.7 \\
\hline & 38 & 7.7 & 1.7 \\
\hline & 22.6 & 15 & 2.15 \\
\hline & 0.9 & 37.9 & 1.55 \\
\hline & 33.3 & 25.6 & 3.1 \\
\hline & 15.8 & 7.88 & 2.46 \\
\hline & 58 & 3.58 & 3.52 \\
\hline Average & 36.1 & 18.1 & 4.0 \\
\hline Maximum percentage error & 63.4 & 37.9 & 8.7 \\
\hline
\end{tabular}

crosstalk noise. Experiments show that our technique is at least $2 \mathrm{X}$ better than the previous works in terms of the accuracy. Experimental results show that the maximum error is less than $10 \%$ and the average error is $4 \%$. We also briefly discussed sizing and buffering techniques for the noise reduction. We used our new metric as a noise calculation engine for these optimizations.

\section{REFERENCES}

[1] L. T. Pillage, R. A. Rohrer, "Asymptotic Waveform Evaluation for Timing Analysis," IEEE. Trans. Computer-Aided Design, vol.9, pp.352-366, Apr. 1990.

[2] P. Feldmann, R. W. Freund, "Efficient Linear Circuit Analysis by Pade Approximation via the Lanczos Process,"IEEE. Trans. Computer-Aided Design, vol. 14, pp.639-649, 1995.

[3] K. J. Kerns, A. T. Yang, "Stable and Efficient Reduction of Large, Mutliport RC Networks by Pole Analysis via Congruence Transformations," IEEE. Trans. Computer-Aided Design, vol. 16, 1997.

[4] A. Odabasioglu, M. Celik, L. T. Pileggi, "PRIMA: Passive Reduced-Order Interconnect Macromodeling Algorithm," IEEE. Trans. Computer-Aided Design, vol. 17, no. 8, pp. 645-654, 1998.
[5] P. Rabiei, M. Pedram, "Model Order Reduction of Large Circuits Using Balanced Truncation," IEEE Proc. ASP-DAC, 1999

[6] A. Vittal, M. Marek-Sadowska, "Crosstalk Reduction for VLSI," IEEE. Trans. Computer-AidedDesign, vol. 16, pp. 290-298, Mar. 1997.

[7] A. Vittal, L. H. Chen, M. Marek-Sadowska, K. P. Wang, S. Yang "Crosstalk in VLSI Interconnections," IEEE. Trans. Computer-Aided Design, vol. 18, no. 12, pp. 1817-1824, 1999.

[8] A. Devgan, "Efficient Coupled Noise Estimation for On-chip Interconnects," IEEE Proc. ICCAD,pp. 147-153. Nov. 1997.

[9] Star-HSPICE Manual, Avanti Corp. , 1999.

[10] C. J. Alpert, A. Devgan, S. T. Quay, "Buffer Insertion for Noise and Delay Optimization," IEEE. Trans. Computer-Aided Design, vol. 18, no. 11, pp. 1633-1645, 1999. 